\title{
ON SEMIGROUP ALGEBRAS AND SEMISIMPLE SEMILATTICE SUMS OF RINGS
}

\author{
by MARK L. TEPLY ${ }^{1}$
}

(Received 17th July 1979)

Let $P$ be a semilattice. In (5), a ring $T$ is called a supplementary semilattice sum of subrings $T_{\alpha}(\alpha \in P)$ if the following conditions hold: $T=\sum_{\alpha \in P} T_{\alpha}, T_{\alpha} T_{\beta} \subseteq T_{\alpha \beta}$ for all $\alpha, \beta \in P$, and $T_{\alpha} \cap\left(\sum_{\alpha \neq \beta} T_{\beta}\right)=0$ for each $\alpha \in P$. Thus, as an abelian group, $T$ is a direct sum of the additive subgroups $T_{\alpha}(\alpha \in P)$, and the multiplicative structure of $T$ is strongly influenced by the semilattice $P$. Properties of these rings have been studied extensively in (2), (3), (5), and (6).

Let $\pi$ be a property of rings. A ring is called a $\pi$-ring if it has property $\pi$. An ideal $I$ of a ring is a $\pi$-ideal if $I$ is a $\pi$-ring. A ring is $\pi$-semisimple if it has no nonzero $\pi$-ideals. Assume that the property $\pi$ satisfies the following conditions: (a) homomorphic images of $\pi$-rings are $\pi$-rings, and (b) ideals of $\pi$-rings are $\pi$-rings. For example, the properties of being nil, nilpotent, left quasi-regular, or von Neumann regular are such properties.

It is known (see (5) and (6)) that if each ring $T_{\alpha}(\alpha \in P)$ is $\pi$-semisimple, then the supplementary semilattice sum $T$ of subrings $T_{\alpha}(\alpha \in P)$ is also $\pi$-semisimple. $\mathrm{J}$. Weissglass has posed the following converse problem (6, Question 1, p. 477): find a condition on the semilattice $P$ such that if $T$ is any supplementary semilattice sum of subrings $T_{\alpha}(\alpha \in P)$, then each $T_{\alpha}(\alpha \in P)$ must be $\pi$-semisimple whenever $T$ is $\pi$-semisimple. By proving a theorem on semigroup rings, we obtain the answer to Weissglass' problem: $\boldsymbol{P}$ must be trivial. (Facts about semigroup rings can be found in (1), (5), and (6).) In particular, if $\pi$ is the property of being nil, nilpotent, or left quasi-regular, Weissglass' problem is answered by setting $T=R S$ and $T_{\alpha}=R S_{\alpha}(\alpha \in P)$ in the following result.

Theorem. Let $P$ be any semilattice with at least 2 elements. If $P$ has a zero (minimal) element, denote it by $\mu$. For any field $R$, there exist semigroups $S_{\alpha}(\alpha \in P)$ such that

(1) $S=\bigcup_{\alpha \in P} S_{\alpha}$ is a semilattice $P$ of semigroups $S_{\alpha}$,

(2) the semigroup ring $R S_{\alpha}$ has a nonzero nilpotent ideal whenever $\alpha \neq \mu$, and

(3) the semigroup ring $R S$ is Jacobson semisimple.

Proof. For each $\alpha \in P$, let $F_{\alpha}$ be the free semigroup without identity on the symbols

$$
\left\{x_{1 \gamma}, x_{2 \gamma}, x_{3 \beta} \mid \gamma \geqq \alpha, \beta>\alpha\right\} \text {. }
$$

\footnotetext{
${ }^{1}$ The author received support from National Science Foundation Grant MCS 77-01818
} 
Let $G_{\alpha}$ be the semigroup obtained by adjoining a zero element $z$ to $F_{\alpha}$. Let $D_{\alpha}$ be the semigroup obtained by imposing the following relations on $G_{\alpha}$ :

(i) $x_{1 \gamma}^{2}=x_{1 \gamma} x_{2 \gamma}=x_{2 \gamma} x_{1 \gamma}=x_{2 \gamma}^{2}$ for all $\gamma \geqq \alpha$, and

(ii) $x_{i \gamma} x_{i \beta}=z=x_{i \beta} x_{i \gamma}$ for $i, j \in\{1,2,3\}$ and $\beta \neq \gamma$.

Define $S_{\alpha}=D_{\alpha}-\left\{x_{1 \gamma}, x_{2 \gamma} \mid \gamma>\alpha\right\}$ for $\alpha \neq \mu$, and define $S_{\mu}=D_{\mu}-\left\{x_{1 \gamma}, x_{2 \gamma} \mid \gamma \geqq \mu\right\}$ whenever $\mu$ exists. Thus $D_{\alpha}$ is an ideal extension of $S_{\alpha}$ for each $\alpha \in P$. Let $\varphi_{\beta \gamma}: S_{\beta} \rightarrow$ $D_{\gamma}$ be the inclusion map whenever $\beta, \gamma \in P$ with $\beta \geqq \gamma$. Then $S=\bigcup_{\alpha \in P} S_{\alpha}$ is a semilattice $P$ of semigroups $S_{\alpha}$ via the homomorphisms $\varphi_{\beta \gamma}$; that is, for $x \in S_{\sigma}$ and $y \in S_{r}, x \cdot y=$ $\left(x \varphi_{\sigma, \sigma \tau}\right)\left(y \varphi_{\tau, \sigma \tau}\right) \in S_{\sigma \tau}$. (See (4, Theorem III.7.2) for details.)

In view of (ii), each element of $D_{\alpha}(\alpha \in P)$ may be written as $z$ or a monomial that is homogeneous in $\gamma \in P$ (i.e., a monomial in $x_{1 \beta}, x_{2 \beta}, x_{3 \beta}$ for some $\beta>\alpha$ or a monomial in $x_{1 \alpha}$ and $\left.x_{2 \alpha}\right)$. We will assume that all elements of $S_{\alpha}$ and $D_{\alpha}(\alpha \in P)$ are written in this form. As usual, the support of an element $t=\sum r_{k} s_{k} \in R S$, denoted by supp $t$, is $\left\{s_{k} \in S \mid r_{k} \neq 0\right\}$.

For any ring $Q$, let $J(Q)$ denote the Jacobson radical of $Q$.

We now establish (2) and (3) by proving a sequence of lemmas.

Lemma 1. For any $\alpha \in P$, the support of any element of $J\left(R S_{\alpha}\right)$ cannot contain a monomial involving an $x_{i \beta}$ for any $\beta>\alpha$ and $i \in\{1,2,3\}$.

Proof. Fix $\beta>\alpha$, and let $B=\left\{x_{i_{1} \beta} x_{i_{2} \beta} \ldots x_{i_{m} \beta} \mid m \geqq 1, i_{j}=1,2\right.$ or 3 for all $\left.j\right\} \subseteq D$. that is, $B$ is the set of all monomials in $D_{\alpha}$ involving an $x_{i \beta}$ entry by (ii). To obtain a contradiction, we assume that $t \in J\left(R S_{\alpha}\right)$ and ( $\left.\operatorname{supp} t\right) \cap B \neq \varnothing$. By (i) and (ii), $\left(\operatorname{supp} x_{3 \beta} t x_{3 \beta}\right) \cap B \neq \varnothing, 0 \neq x_{3 \beta} t x_{3 \beta} \in J\left(R S_{\alpha}\right)$, and the only monomials in supp $x_{3 \beta} t x_{3 \beta}$ that have degree $>1$ start and end with $x_{3 \beta}$. Choose $t^{\prime} \in R S_{\alpha}$ such that

$$
x_{3 \beta} t x_{3 \beta}+t^{\prime}+x_{3 \beta} t x_{3 \beta} t^{\prime}=0 \text {. }
$$

If $\quad\left(\operatorname{supp} t^{\prime}\right) \cap B=\varnothing$, then $\left(\operatorname{supp} x_{3 \beta} t x_{3 \beta} t^{\prime}\right) \cap B=\varnothing \quad$ by (ii). But then $B \cap \operatorname{supp}\left(x_{3 \beta} t x_{3 \beta}+t^{\prime}+x_{3 \beta} t x_{3 \beta} t^{\prime}\right) \neq \varnothing$, which contradicts the fact that $x_{3 \beta} t x_{3 \beta}+t^{\prime}+$ $x_{3 \beta} t x_{3 \beta} t^{\prime}=0$. Hence $\left(\operatorname{supp} t^{\prime}\right) \cap B \neq \varnothing$.

By (i) we assume that each member of $B$ is written with as many $x_{1 \beta}$ entries as possible (and hence as few $x_{2 \beta}$ entries as possible). Then we can order $B$ as follows:

$$
x_{i_{1} \beta} x_{j_{2} \beta} \ldots x_{i_{m} \beta}>x_{k_{1} \beta} x_{k_{2} \beta} \ldots x_{k_{n} \beta}
$$

if either (a) $m>n$ or else (b) $m=n, j_{1}=k_{1}, j_{2}=k_{2}, \ldots, j_{q-1}=k_{q-1}, j_{q}>k_{q}$ for some $q \leqq n$. Thus, if $e, f, g, h \in B$ such that $e>f, g>h$, and both $e$ and $f$ end in $x_{3 \beta}$, then $e g>f g$ and $e g>e h$. Let $e$ and $g$ be maximal in $\left(\operatorname{supp} x_{3 \beta} t x_{3 \beta}\right) \cap B$ and $\left(\operatorname{supp} t^{\prime}\right) \cap B$, respectively. Then $e g \in \operatorname{supp} x_{3 \beta} t x_{3 \beta} t^{\prime}$, and $e g \notin\left(\operatorname{supp} x_{3 \beta} t x_{3 \beta}\right) \cup\left(\operatorname{supp} t^{\prime}\right)$. This contradicts the assumption that $x_{3 \beta} t x_{3 \beta}+t^{\prime}+x_{3 \beta} t x_{3 \beta} t^{\prime}=0$.

Lemma 2. For any $\alpha \in P, r z \in J\left(R S_{\alpha}\right)$ implies $r=0$.

Proof. The ideal $R z$ of $R S_{\alpha}$ is (ring) isomorphic to the field $R$; so $J\left(R S_{\alpha}\right) \cap R z=$ $J(R z)=0$. 
Lemma 3. $J\left(R S_{\mu}\right)=0$ if $\mu$ exists, and $J\left(R S_{\alpha}\right)=\left\{r x_{2 \alpha}-r x_{1 \alpha} \mid r \in R\right\}$ is a nilpotent ideal of $R S_{\alpha}$ for $\alpha \neq \mu$.

Proof. Let $\alpha \in P$, and let $H$ be the ideal of $R D_{\alpha}$ generated by the set

$$
\left\{r z, r x_{1 \alpha}, r x_{2 \alpha} \mid r \in R\right\} \text {. }
$$

By Lemma 1, $J\left(R S_{\alpha}\right) \subseteq H$. Hence $J\left(R S_{\alpha}\right)=J\left(R S_{\alpha}\right) \cap\left(H \cap R S_{\alpha}\right)=J\left(H \cap R S_{\alpha}\right)=$ $\left(H \cap R S_{\alpha}\right) \cap J(H) \subseteq J(H)$. The mapping $\theta: H \rightarrow R[x]$ given by

$$
\left(a z+b x_{2 \alpha}+\sum_{i=1}^{n} c_{i} x_{1 \alpha}^{i}\right) \theta=b x+\sum_{i=1}^{n} c_{i} x^{i}
$$

is a ring homomorphism of $H$ onto the polynomial ring $R[x]$. (Note that if $\alpha=\mu$, then $b=c_{1}=0$.) Thus $(J(H)) \theta \subseteq J(R[x])=0$; so

$$
J\left(R S_{\alpha}\right) \subseteq J(H) \subseteq \operatorname{ker} \theta=\left\{a z+b x_{2 \alpha}-b x_{1 \alpha} \mid a, b \in R\right\} .
$$

If $\alpha=\mu$, then $b=0$, and hence $J\left(R S_{\mu}\right)=0$ by Lemma 2 .

Assume now that $\alpha \neq \mu$, and let $N_{\alpha}=\left\{r x_{2 \alpha}-r x_{1 \alpha} \mid r \in R\right\}$. If $a z+b x_{2 \alpha}-b x_{1 \alpha} \in J\left(R S_{\alpha}\right)$, then $a z=\left(a z+b x_{2 \alpha}-b x_{1 \alpha}\right) x_{1 \alpha} \in J\left(R S_{\alpha}\right)$; so $a=0$ by Lemma 2 . Hence $J\left(R S_{\alpha}\right) \subseteq N_{\alpha}$. But a straightforward computation using (i) and (ii) shows that $\left(R S_{\alpha}\right) N_{\alpha}=0=N_{\alpha}\left(R S_{\alpha}\right)$. Thus $N_{\alpha}$ is a nonzero nilpotent ideal of $R S_{\alpha}$, and hence $N_{\alpha} \subseteq J\left(R S_{\alpha}\right)$.

For $t=\sum_{\alpha \in \mathbf{P}} t_{\alpha} \in R S$ with $t_{\alpha} \in R S_{\alpha}$, let

$$
P \text {-supp } t=\left\{\alpha \in P \mid t_{\alpha} \neq 0\right\},
$$

and

$\max P$-supp $t=\{\alpha \in P$-supp $t \mid \beta \in P$-supp $t$ and $\beta \geqq \alpha$ imply $\beta=\alpha\}$.

Lemma 4. $J(R S)=0$.

Proof. To obtain a contradiction, assume that $0 \neq t=\sum_{\alpha \in P} t_{\alpha} \in J(R S)$ with $t_{\alpha} \in R S_{\alpha}$. Let $\beta \in \max P$-supp $t$. As in the proof of (5, Theorem 1), $0 \neq t_{\beta} \in J\left(R S_{\beta}\right)$. By Lemma 3, $\beta \neq \mu$ and $t_{\beta}=r x_{2 \beta}-r x_{1 \beta} \in R S_{\beta}$ for some nonzero $r \in R$. Let $\gamma<\beta$; for $x_{3 \beta} \in R S_{\gamma}$, write $x_{3 \beta} \cdot t=\sum_{\alpha \in P} t_{\alpha}^{\prime}$. Then $t_{\gamma}^{\prime}=r x_{3 \beta} x_{2 \beta}-r x_{3 \beta} x_{1 \beta}+a z+$ terms whose support consists of monomials of degree at least two in either $x_{1 \beta}$ or $x_{3 \beta}$. (The terms after the first two may be 0 .) But $\{\gamma\}=\max P$-supp $x_{3 \beta} t$ for $x_{3 \beta} \in R S_{\gamma}$. Again, as in the proof of $(5$, Theorem 1), $t_{\gamma}^{\prime} \in J\left(R S_{\gamma}\right)$. Hence our computed form of $t_{\gamma}^{\prime}$ contradicts Lemma 3 . This completes the proof of the Theorem.

Remark. The Theorem of this paper shows that conditions on a non-trivial semilattice $P$ alone are not sufficient for the $\pi$-semisimplicity of the semilattice sum $T$ of subrings $T_{\alpha}(\alpha \in P)$ to force each $T_{\alpha}$ to be $\pi$-semisimple. In particular, additional restrictions must be placed on $T$ to ensure the transfer of $\pi$-semisimplicity to each $T_{\alpha}$. We have seen that requiring $T$ to be a semigroup ring $R S$, where $S$ is a semilattice of semigroups $S_{\alpha}$, is also not sufficient; the problem arises because the images of the homomorphisms $\left\{\varphi_{\alpha, \beta} \mid \alpha, \beta \in P, \alpha \geqq \beta\right\}$ are not in $S_{\beta}$. In case the images of the defining 
homomorphisms $\varphi_{\alpha \beta}$ are always in $S_{\beta}(\beta \in P)$, then $S$ is called a strong semilattice $P$ of semigroups $S_{\alpha}(\alpha \in P)(4)$; for this case conditions on $P$ have been found to ensure the transfer of $\pi$-semisimplicity from $R S$ to each $R S_{\alpha}$ (see (5, Theorem 2)). In particular, the Theorem of this paper shows that the "strong" hypothesis on $S$ cannot be dropped in (5, Theorem 2).

Another way to ensure the transfer of $\pi$-semisimplicity from the semilattice sum $T$ to each $T_{\alpha}(\alpha \in P)$ is to place additional restrictions on the property $\pi$. As a consequence (2, Theorem 1), $\pi$-semisimplicity transfers from $T$ to each $T_{\alpha}(\alpha \in P)$ when either of the following conditions holds: (a) $\pi$ is a strict, hereditary radical property and $P$ is finite, or (b) $\pi$ is an $A$-radical property. (See (2) for a discussion of the strong conditions on $\pi$ in (a) and (b).) It is not known if the condition that $P$ is finite can be removed from (a).

\section{REFERENCES}

(1) A. H. Clifford and G. B. Preston, Algebraic Theory of Semigroups, Vol. I. (Math. Surveys of the Amer. Math. Soc., Providence, R. I., 1961).

(2) B. J. GARDNER, Radicals of supplementary semilattice sums of associative rings, Pacific $J$. Math. 58 (1975), 387-392.

(3) J. JANESKI and J. WeIssglass, Regularity of semilattice sums of rings, Proc. Amer. Math. Soc. 39 (1973), 479-482.

(4) M. Petrich, Introduction to Semigroups (C. Merrill, Columbus, Ohio, 1973).

(5) M. L. Teply, E. G. Turman, and A. Quesada, On Semisimple semigroup Rings, Proc. Amer. Math. Soc. 79 (1980), 157-163.

(6) J. Weissglass, Semigroup Rings and Semilattice Sums of Rings, Proc. Amer. Math. Soc. 39 (1973), 471-478.

UNIVERSITY OF FLORIDA

Gainesville, Florma 32611

U.S.A. 